J Pediatr. 2018 January ; 192: 204-208. doi:10.1016/j.jpeds.2017.09.003.

\title{
Newborn Screening in the United States May Miss Mild Persistent Hypothyroidism
}

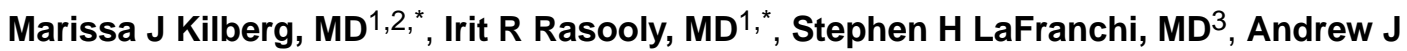 \\ Bauer, MD ${ }^{2,4}$, and Colin P Hawkes, MD ${ }^{1,2}$ \\ ${ }^{1}$ Department of Pediatrics, The Children's Hospital of Philadelphia, Philadelphia, USA \\ 2Division of Endocrinology and Diabetes, The Children's Hospital of Philadelphia, Philadelphia, \\ USA \\ ${ }^{3}$ Pediatric Endocrinology, Oregon Health and Science University, Portland, Oregon, USA \\ ${ }^{4}$ Department of Pediatrics, Perelman School of Medicine, University of Pennsylvania, \\ Philadelphia, USA
}

\begin{abstract}
Objective-To determine if newborn screening (NBS) programs for congenital hypothyroidism in the USA utilize TSH cutoffs that are age-adjusted to account for the physiologic four-fold reduction in TSH concentrations over the first few days of life.
\end{abstract}

Study design-All NBS programs in the USA were contacted and asked to provide information on their NBS protocols, TSH cutoffs and whether these cutoffs were age-adjusted.

Results-Of 51 NBS programs, 28 request a repeat specimen if the initial eluted serum TSH concentration is mildly increased (between the cutoff and a median upper limit of $50 \mathrm{mU} / \mathrm{L}$ ), while 14 programs perform a routine second screen in all infants. Although these specimens are typically collected between 1 week and 1 month of life, 16 of the 28 programs with a discretionary second test and 8 of 14 programs with a routine second test do not have age-adjusted TSH cutoffs after the first 48 hours of life.

Conclusions-There is variation in NBS practices for screening for $\mathrm{CH}$ across the USA and many programs do not adjust the TSH cutoff beyond the first two days of life. Samples are processed when received from older infants, often to retest borderline initial results. This approach will miss $\mathrm{CH}$ in infants with persistent mild TSH elevations. We recommend that all NBS programs provide age-adjusted TSH cutoffs, and suggest developing a standard approach to screening for $\mathrm{CH}$ in the USA.

Address correspondence to: Dr. Colin P Hawkes, Division of Endocrinology and Diabetes, The Children's Hospital of Philadelphia, Philadelphia, PA, USA., Telephone: +1 215-590-3174, Fax: +1 215-590-3053, hawkesc@email.chop.edu.

contributed equally

The authors declare no conflicts of interest.

Publisher's Disclaimer: This is a PDF file of an unedited manuscript that has been accepted for publication. As a service to our customers we are providing this early version of the manuscript. The manuscript will undergo copyediting, typesetting, and review of the resulting proof before it is published in its final citable form. Please note that during the production process errors may be discovered which could affect the content, and all legal disclaimers that apply to the journal pertain. 


\section{Keywords}

congenital hypothyroidism; newborn screen; thyroid; newborn; TSH; USA; United States; cutoff

Congenital hypothyroidism has an incidence of approximately 1 in 2000 to 4000 births $^{1}$ and over 1400 of the 3.4 million infants born in the United States of America (USA) are diagnosed with $\mathrm{CH}$ annually ${ }^{2}$. Prior to the universal implementation of newborn screening (NBS), $\mathrm{CH}$ was a leading cause of intellectual disability ${ }^{2}$. The prevalence of $\mathrm{CH}$ has also increased with the implementation of universal screening, demonstrating detection of milder $\mathrm{CH}$ that was not associated with severe symptomatic intellectual disability ${ }^{3}$.

The critical role of thyroid hormone in normal development and growth is supported by the positive effect of early identification and treatment of $\mathrm{CH}$ on neurodevelopmental outcomes $^{4,5}$. Even when $\mathrm{CH}$ is identified early, disease severity at the time of diagnosis and the time to normalization of thyroid stimulating hormone (TSH) may affect cognitive outcome $^{6,7}$. Persistent moderate increases in TSH concentrations can be seen in children without severe $\mathrm{CH}$. This includes infants with thyroid hypoplasia, hemiagenesis, ectopy or normally appearing thyroid glands that may ultimately develop into a goiter secondary to disorders involving thyroid dyshormonogenesis (eg, iodine organification defects, Pendred syndrome, defective thyroglobulin synthesis, a defective sodium-iodine symporter, pseudohypoparathyroidism or trisomy 21$)^{8}$.

The early detection of severe $\mathrm{CH}$ prior to the development of symptoms can significantly reduce the likelihood of developing intellectual disability. ${ }^{9}$ The effect of untreated isolated mild hyperthyrotropinemia is likely to be more subtle, and it remains controversial regarding whether very mild persistent elevations in TSH concentrations have adverse neurodevelopmental consequences. At the lower end of this spectrum, infants with whole blood TSH concentrations of up to $15 \mathrm{mU} / \mathrm{L}$ do not have detectable reductions in intelligence quotient (IQ) ${ }^{10}$ or psychomotor development ${ }^{11}$. In studies showing neurocognitive effects of mild hyperthyrotropinemia, confounding effects of maternal iodine deficiency on fetal development may reduce the generalizability of any benefit of treatment to iodine-sufficient populations ${ }^{12,13}$. A large study in Australia describing cognitive outcomes in children with mild elevations in TSH concentrations on NBS describes an association between impairment in cognitive outcome with elevations in TSH concentration, but this may also be confounded by maternal iodine status ${ }^{14}$. Consequently, guidelines from the European Society for Paediatric Endocrinology recommend observing mild increases in venous TSH concentrations for up to $3-4$ weeks before considering initiating treatment ${ }^{15}$. Once treatment is initiated, close follow-up is recommended and reevaluation at age 3 years is recommended if imaging does not show a permanent form of $\mathrm{CH}$, or if there is no rise in TSH concentration above $10 \mathrm{mU} / \mathrm{L}$ after 1 year ${ }^{16,17}$.

Following delivery, serum TSH concentrations in a term infant rise to a mean of $\sim 80 \mathrm{mU} / \mathrm{L}$ at 30 minutes of life and then decrease rapidly over the next two days ${ }^{18}$; TSH concentration is usually $<10 \mathrm{mU} / \mathrm{L}$ at one week of life and is typically within the normal range for children and adults by two weeks of age ${ }^{19}$. This normal transitional pattern is the basis for establishing age-based specific norms for newborn TSH concentrations within the first week 
of life. Although these higher TSH concentrations are considered normal during the first 48 to 72 hours of life, similar elevations in TSH outside of this time frame may be a sign of underlying thyroid dysfunction. ${ }^{19,}{ }^{20}$ Recognizing this, guidelines from the American Academy of Pediatrics recommend that "...blood be collected when the infant is between 2 and 4 days of age," 2 and guidelines from the European Society for Pediatric Endocrinology state "...the best "window' for testing is 48 to 72 hours of age." 15 Thus, if a NBS sample is collected outside of the recommended timeframe, an infant with persistent mild increased TSH concentrations would be misclassified as having a normal result if reference ranges do not account for the age of the infant at the time of sample collection.

The aims of this study were to describe the approaches to NBS for CH across the USA, and to determine if these screening algorithms use age-specific cutoffs for TSH concentrations in term infants.

\section{METHODS}

All newborn screening programs in the USA were included in this study. Laboratories and departments of public health were contacted by phone initially, or email if the personnel were not available. Where possible, information was supported by review of www.newsteps.org or published protocols.

Data were collected using semi-structured interviews inquiring about modality of testing, recommended age of testing and age limits of newborn screens, TSH cutoffs (including cutoff for action), and protocols for handling abnormal results. Only protocols pertaining to term infants were included. All NBS programs in the USA report screening TSH results as serum concentrations. Because most programs assume an average hematocrit of 50-55\% in newborns, whole blood TSH concentrations are half the equivalent serum concentration. When comparing our results with studies that report whole blood TSH concentrations, whole blood values were multiplied by 2 to calculate comparable serum TSH concentrations.

As patient information was not collected or analyzed, the Institutional Review Board at The Children's Hospital of Philadelphia deemed this study exempt from review.

\section{Statistical Analyses}

Data were categorized by testing protocol (primary TSH with backup thyroxine (T4), combined primary TSH \& T4, primary T4 with backup TSH), whether or not a routine repeat NBS is performed, age-specific TSH cutoffs, how many times TSH reference values are age adjusted, whether confirmatory serum or repeat NBS are recommended or accepted for borderline elevated TSH, and the age limit at which repeat NBS are no longer accepted. In programs that utilized initial T4 testing, the TSH cutoffs were used for analysis. T4 cutoffs were not analyzed. Only TSH levels beyond the first 24 hours of life (i.e. the recommended screening period) were analyzed.

Analyses were conducted in SPSS version 22.0 (IBM, New York, USA). Figures were generated using Prism 5.0 (GraphPad Software Inc, California) and Adobe Illustrator (Adobe Systems Inc, California). 


\section{RESULTS}

All 51 NBS programs in the USA were contacted and representatives from 50 programs provided information through semi-structured interview. Information regarding the process in the remaining program was available online.

Among healthy, term infants the NBS collection is recommended in the first one to three days of life in all programs. Despite this recommendation, 50 of the 51 programs process initial NBS samples beyond the first 4 days of life. One program recommends that physicians measure serum TSH concentrations (rather than process a late NBS) if the initial window for NBS is missed; 14 programs (27\%) perform a routine second NBS for all newborns between one week and six weeks of life.

Twenty-two (43\%) programs measure primary TSH concentration. Of these, 6 subsequently measure total T4 concentrations in abnormal samples and 16 (31\%) have algorithms based solely on TSH measurements. Twenty programs (39\%) measure primary T4 and measure TSH concentrations only if T4 is abnormal. Nine (18\%) programs measure both TSH and T4 in all newborns (Figure 1).

All programs have a TSH level which triggers immediate clinical referral and serum thyroid function testing.

For borderline elevated TSH concentrations (defined as TSH concentrations between the cutoff for normal results, but below the cutoff for recommendation of urgent serum testing), nine programs recommend obtaining serum thyroid function testing. These nine programs do not recommend repeat NBS. Fourteen programs perform routine repeat NBS (which includes TSH) for all infants and, thus, no additional testing is recommended for borderline increased TSH. In addition, eight of these programs use the same TSH cutoff for the initial and repeat NBS despite the infant being older at the time of repeat testing.

In 28 programs, repeat NBS are requested for borderline increased TSH concentrations. Of these, 12 measure only TSH concentrations on the repeat specimen. In these programs, the median TSH concentration above which urgent serum testing is recommended instead of repeat NBS is $50 \mathrm{mU} / \mathrm{ml}$ (IQR 50-60). In the other 16 of 28 programs that request repeat NBS, there are a combination of primary TSH with reflex to T4 and primary T4 with reflex to TSH testing. These programs have varied algorithms for referral and incorporate extremely low T4 concentrations as triggers for confirmatory serum testing.

TSH cutoffs were available from 49 of the 50 programs that accept and process thyroid testing on NBS received beyond the first 72 hours of life. Of these, 24 do not adjust the TSH cutoff according to the age of the infant. In these programs, the TSH concentration for an abnormal test (after 24 hours of life) ranges from $20-40 \mathrm{mU} / \mathrm{ml}$ with the median (IQR) of 25 (20-30) $\mathrm{mU} / \mathrm{ml}$ regardless of age of infant at the time of the test. Additionally, each program has a cutoff value above which immediate treatment and or referral to endocrinology is recommended (median (IQR) of $50(40-60) \mathrm{mU} / \mathrm{ml})$. The reported upper limit of normal for TSH concentrations on NBS according to the infant's age for each program is shown in Figures 2 and 3. This demonstrates that when NBS tests are performed outside of the first 
few days of life, TSH concentration cutoffs far higher than the age-appropriate range may be reported as normal in many programs.

Included in these 24 programs that do not adjust TSH concentration cutoffs according to the infant's age are 16 programs that request repeat NBS (rather than serum testing) for borderline increased TSH. Thus, the cutoff for TSH concentrations to be reported as abnormal in these 16 programs for the repeated test in an older infant is the same as for those within the first three days of life. Similarly, eight of the 14 programs that perform routine second NBS for all infants do not adjust the TSH cutoff concentration for the second test.

\section{DISCUSSION}

There is variation in practice amongst NBS programs across the USA in the approach to screening for $\mathrm{CH}$. The key finding of this study is that many programs do not adjust the reported TSH cutoffs according to the infant's age, despite adopting protocols that may require repeat NBS TSH measurements outside of the age for which the cutoffs were established. In programs that adjust TSH cutoffs according to age, most do not adjust beyond day three of life despite accepting samples up to and beyond one month of age.

Based on the data presented in this study, an infant with a serum TSH concentration of 24 $\mathrm{mU} / \mathrm{ml}$ will be incorrectly reported as "normal" in 28 programs if the sample was collected and run on a NBS platform on day of life four, and 21 programs on day of life seven. This would affect infants with delayed initial NBS and infants with a recommended repeat NBS submitted because of a borderline elevated TSH on the initial NBS. Only four programs would flag a serum TSH concentration of $14 \mathrm{mU} / \mathrm{ml}$ on day ten of life as abnormal. Although infants with normal thyroid function have a four-fold decline in TSH concentrations between the first and the fourth day of life ${ }^{8}$, this may not fall to normal ranges in children with mild persistent $\mathrm{CH}^{21,22}$. NBS protocols that do not apply agespecific TSH cutoff concentrations will not identify these infants.

There is active discussion surrounding the cost to benefit ratio to lowering the TSH cutoffs for NBS. In Greece, a lowering of the whole blood TSH cutoff for recall at day three to five from 20 to $10 \mathrm{mU} / \mathrm{ml}$ (i.e. serum TSH concentration from 40 to $20 \mathrm{mU} / \mathrm{ml}$ ) increased the recall rate from 0.12 to $1.2 \%$ and, over $70 \%$ of the additional patients diagnosed with $\mathrm{CH}$ following this change were found to have permanent disease ${ }^{22}$. Similar changes to the TSH cutoff in Quebec did not identify additional infants with severe $\mathrm{CH}$, but over $70 \%$ of additional infants identified had permanent primary hypothyroidism ${ }^{23}$. Although any reduction in TSH cutoffs increases the recall rate, costs, and parental anxiety, approximately one third of infants with screening whole blood TSH concentrations between 16 and 20 $\mathrm{mU} / \mathrm{ml}$ (ie, between serum TSH concentration of 32 to $40 \mathrm{mU} / \mathrm{ml}$ ) have persistent hypothyroidism ${ }^{21,22}$. However, it is not known what the effect of applying age-appropriate TSH cutoffs to NBS samples collected outside the age window for which the normative data was established would be on recall rate. 
The main strengths of this study are that it is a comprehensive review of NBS protocols for $\mathrm{CH}$ screening in healthy, term infants older than 24 hours in the USA, and it identifies a correctable short-fall in the current approach to NBS in several States. Many programs utilize complex algorithms for identifying children with $\mathrm{CH}$ and assess both TSH and T4; nonetheless, absent or insufficient age-adjustment of TSH cutoffs on NBS appears to be a common issue regardless of which algorithm is used. In an effort to avoid bias, or an exaggeration in the potential risk, we utilized the lowest TSH cutoffs described and may have slightly underestimated the risk. The limitation of this study is that we were unable to determine the direct clinical impact of these variable approaches to NBS in the USA. Without systematically following up patients who pass NBS according to current Statespecific algorithms or data on the diagnosis of hypothyroidism within the first few years of life, it was not possible to study the prevalence of missed persistent $\mathrm{CH}$ in this population. Is would also be difficult to prospectively study this through prospectively following infants with persistent hyperthyrotropinemia to assess potential negative neurocognitive outcomes, as current guidelines ${ }^{15}$ indicate that there would not be equipoise to withhold levothyroxine treatment in this context.

In conclusion, we have demonstrated significant variation in the approach to NBS for $\mathrm{CH}$ across the USA. We have shown that high TSH cutoffs, validated for first three days of life, are often used to assess thyroid function in infants older than 72 hours of life. We recommend that all programs consider using age-adjusted TSH cutoffs, given that current approaches may miss children with mild persistent $\mathrm{CH}$. We also suggest that NBS screening for $\mathrm{CH}$ be reviewed on a national level so that a more standardized approach may be adopted across the USA, although budget implications of any change would have to be approved at the state level.

\section{Acknowledgments}

I.R.R. is supported by the Eunice Kennedy Shriver National Institute of Child Health \& Human Development of the National Institutes of Health (T32HD060550).

\section{Abbreviations}

CH congenital hypothyroidism

IQ Intelligence Quotient

NBS newborn screening

TSH thyroid stimulating hormone

USA United States of America

\section{References}

1. Harris KB, Pass KA. Increase in congenital hypothyroidism in New York State and in the United States. Mol Genet Metab. 2007; 91:268-77. [PubMed: 17512233]

2. Rose SR, Brown RS, Foley T, Kaplowitz PB, Kaye CI, Sundararajan S, et al. Update of newborn screening and therapy for congenital hypothyroidism. Pediatrics. 2006; 117:2290-303. [PubMed: 16740880] 
3. Alm J, Hagenfeldt L, Larsson A, Lundberg K. Incidence of congenital hypothyroidism: retrospective study of neonatal laboratory screening versus clinical symptoms as indicators leading to diagnosis. Br Med J (Clin Res Ed). 1984; 289:1171-5.

4. Albert BB, Heather N, Derraik JG, Cutfield WS, Wouldes T, Tregurtha S, et al. Neurodevelopmental and body composition outcomes in children with congenital hypothyroidism treated with high-dose initial replacement and close monitoring. J Clin Endocrinol Metab. 2013; 98:3663-70. [PubMed: 23861458]

5. Leger J. Congenital hypothyroidism: a clinical update of long-term outcome in young adults. European journal of endocrinology /European Federation of Endocrine Societies. 2015; 172:R6777.

6. Huo K, Zhang Z, Zhao D, Li H, Wang J, Wang X, et al. Risk factors for neurodevelopmental deficits in congenital hypothyroidism after early substitution treatment. Endocr J. 2011; 58:355-61. [PubMed: 21467693]

7. Selva KA, Harper A, Downs A, Blasco PA, Lafranchi SH. Neurodevelopmental outcomes in congenital hypothyroidism: comparison of initial T4 dose and time to reach target T4 and TSH. J Pediatr. 2005; 147:775-80. [PubMed: 16356430]

8. Gaudino R, Garel C, Czernichow P, Leger J. Proportion of various types of thyroid disorders among newborns with congenital hypothyroidism and normally located gland: a regional cohort study. Clin Endocrinol (Oxf). 2005; 62:444-8. [PubMed: 15807875]

9. Grosse SD, Van Vliet G. Prevention of intellectual disability through screening for congenital hypothyroidism: how much and at what level? Arch Dis Child. 2011; 96:374-9. [PubMed: 21242230]

10. Trumpff C, De Schepper J, Vanderfaeillie J, Vercruysse N, Van Oyen H, Moreno-Reyes R, et al. Thyroid-Stimulating Hormone (TSH) Concentration at Birth in Belgian Neonates and Cognitive Development at Preschool Age. Nutrients. 2015; 7:9018-32. [PubMed: 26540070]

11. Trumpff C, De Schepper J, Vanderfaeillie J, Vercruysse N, Van Oyen H, Moreno-Reyes R, et al. Neonatal thyroid-stimulating hormone concentration and psychomotor development at preschool age. Arch Dis Child. 2016; 101:1100-6. [PubMed: 27402733]

12. Calaciura F, Mendorla G, Distefano M, Castorina S, Fazio T, Motta RM, et al. Childhood IQ measurements in infants with transient congenital hypothyroidism. Clin Endocrinol (Oxf). 1995; 43:473-7. [PubMed: 7586623]

13. Azizi F, Afkhami M, Sarshar A, Nafarabadi M. Effects of transient neonatal hyperthyrotropinemia on intellectual quotient and psychomotor performance. Int J Vitam Nutr Res. 2001; 71:70-3. [PubMed: 11276926]

14. Lain SJ, Bentley JP, Wiley V, Roberts CL, Jack M, Wilcken B, et al. Association between borderline neonatal thyroid-stimulating hormone concentrations and educational and developmental outcomes: a population-based record-linkage study. The lancet Diabetes \& endocrinology. 2016; 4:756-65. [PubMed: 27453174]

15. Leger J, Olivieri A, Donaldson M, Torresani T, Krude H, van Vliet G, et al. European Society for Paediatric Endocrinology consensus guidelines on screening, diagnosis, and management of congenital hypothyroidism. Horm Res Paediatr. 2014; 81:80-103. [PubMed: 24662106]

16. Kemper AR, Ouyang L, Grosse SD. Discontinuation of thyroid hormone treatment among children in the United States with congenital hypothyroidism: findings from health insurance claims data. BMC pediatrics. 2010; 10:9. [PubMed: 20156344]

17. Korzeniewski SJ, Grigorescu V, Kleyn M, Young WI, Birbeck G, Todem D, et al. Transient hypothyroidism at 3-year follow-up among cases of congenital hypothyroidism detected by newborn screening. J Pediatr. 2013; 162:177-82. [PubMed: 22878110]

18. Fisher DA, Odell WD. Acute release of thyrotropin in the newborn. The Journal of clinical investigation. 1969; 48:1670-7. [PubMed: 5822577]

19. Lem AJ, de Rijke YB, van Toor H, de Ridder MA, Visser TJ, Hokken-Koelega AC. Serum thyroid hormone levels in healthy children from birth to adulthood and in short children born small for gestational age. J Clin Endocrinol Metab. 2012; 97:3170-8. [PubMed: 22736771] 
20. Williams FL, Simpson J, Delahunty C, Ogston SA, Bongers-Schokking JJ, Murphy N, et al. Developmental trends in cord and postpartum serum thyroid hormones in preterm infants. J Clin Endocrinol Metab. 2004; 89:5314-20. [PubMed: 15531476]

21. Jones JH, Smith S, Dorrian C, Mason A, Shaikh MG. Permanent congenital hypothyroidism with blood spot thyroid stimulating hormone $<10 \mathrm{mU} / \mathrm{L}$. Arch Dis Child. 2016

22. Mengreli C, Kanaka-Gantenbein C, Girginoudis P, Magiakou MA, Christakopoulou I, GiannouliaKarantana A, et al. Screening for congenital hypothyroidism: the significance of threshold limit in false-negative results. J Clin Endocrinol Metab. 2010; 95:4283-90. [PubMed: 20591982]

23. Deladoey J, Ruel J, Giguere Y, Van Vliet G. Is the incidence of congenital hypothyroidism really increasing? A 20-year retrospective population-based study in Quebec. J Clin Endocrinol Metab. 2011; 96:2422-9. [PubMed: 21632812] 


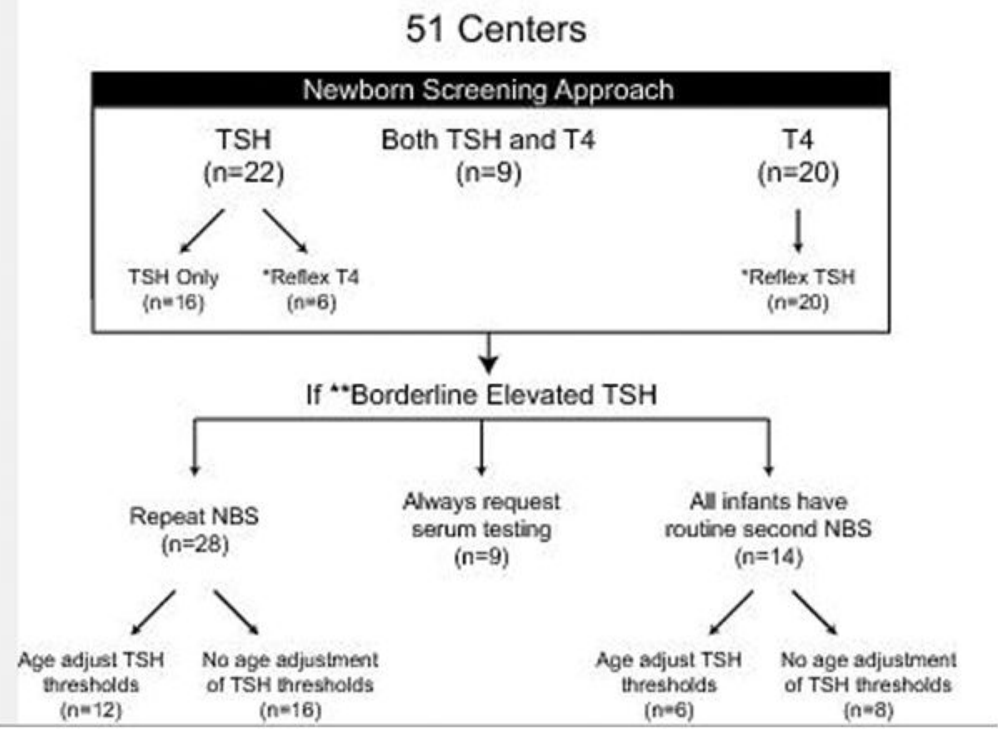

Figure 1.

Approach to newborn screening for congenital hypothyroidism across the United States of America.

* TSH or T4 testing on original sample is subsequently performed if initial T4 or TSH test was abnormal.

** TSH concentrations between the cutoff for normal results, but below the cutoff for direct action (median (IQR) of $50(50-60) \mathrm{mU} / \mathrm{ml})$.

NBS=Newborn Screen, $\mathrm{TSH}=$ thyroid stimulating hormone, $\mathrm{T} 4=$ thyroxine . 


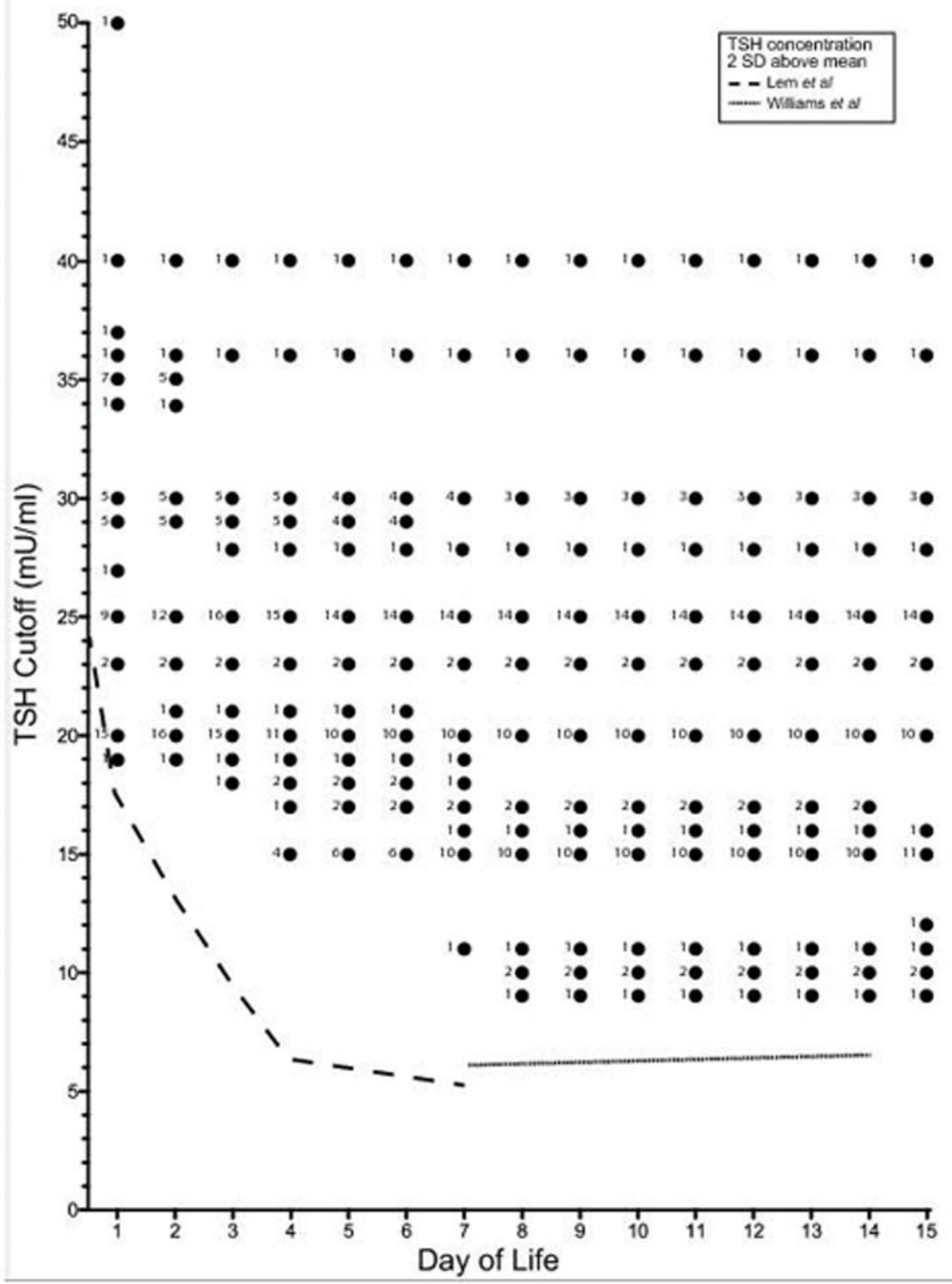

Figure 2.

The cutoff serum TSH concentration on NBS that is categorized as abnormal by infant age in days. Each dot represents the upper limit of normal TSH concentrations at the infant's day of life, and the number designates the number of programs that use this cutoff. Data were not available for one of the 51 programs in the USA, and this program is not represented.

Reference TSH values are reported up until the age that each particular program accepts NBS/repeat TSH. The dashed line represents the two standard deviations above the mean $\mathrm{TSH}$ concentration for term infants at each day of life (Adapted from Lem et al ${ }^{19}$ and Williams et $\mathrm{al}^{20}$ ). 


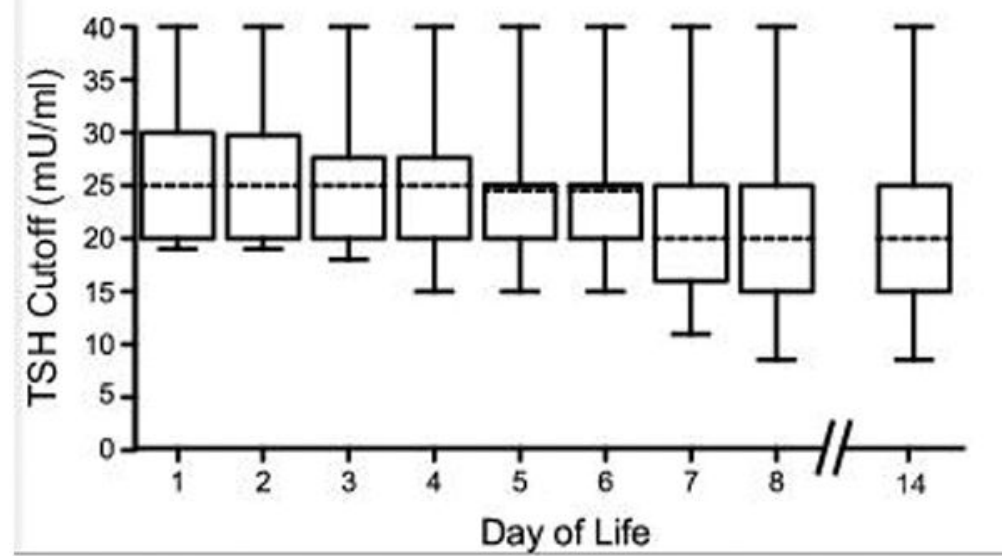

Figure 3.

Range of TSH cutoffs for all NBS programs at various days of life. The bar in each box represents the median cutoff of available programs with the box representing the interquartile range. Of note, 2 states use combined primary TSH and T4 to determine whether or not to repeat the NBS and, thus, were excluded from this figure. 